\title{
De ville modèle à quartier défavorisé : La trajectoire de développement de Kénogami
}

\author{
Martin Simard ${ }^{1}$, Suzanne Tremblay ${ }^{2}$ et Carl Brisson ${ }^{3}$ \\ Université du Québec à Chicoutimi
}

\section{INTRODUCTION}

Le Québec comporte de nombreuses villes

modèles aménagées et développées par des entreprises forestières ou minières, principalement en milieu périphérique. Fermont, Schefferville ou Témiscamingue en sont des exemples bien connus.

Le Québec comporte de nombreuses villes modèles aménagées et développées par des entreprises forestières ou minières, principalement en milieu périphérique. Fermont, Schefferville ou Témiscamingue en sont des exemples bien connus. La région du Saguenay-LacSaint-Jean compte plusieurs «villes de compagnies » ou Company Towns, notamment Arvida, Dolbeau, Port-Alfred et Riverbend. Kénogami est aussi l'une de ces villes modèles. Cette ancienne municipalité autonome qui a été regroupée administrativement avec Jonquière (1975) puis Saguenay (2002) est aujourd'hui un secteur à fort potentiel patrimonial. Kénogami fut fondée en 1912 par les Price Brothers autour d'une papeterie toujours en fonction. Kénogami constitue maintenant un véritable quartier ancien dans la partie ouest de l'agglomération urbaine $\mathrm{du}$ Haut-Saguenay. Le quartier vit de nombreuses difficultés socioéconomiques depuis plus d'un quart de siècle.

Le secteur de Kénogami est enclavé entre les rives escarpées des rivières aux Sables et Saguenay et le tissu urbain relativement dense de Jonquière. Celui-ci connaît un processus continu de paupérisation. Contrairement à sa voisine Arvida, Kénogami n'a pas été en mesure d'utiliser sa forme urbaine particulière pour se mettre en valeur et attirer résidants et industries. De plus, le commerce sur rue, naguère prospère, ressemble étrangement à celui des artères commerciales dévitalisées des métropoles, avec moult locaux vacants et bâtiments décrépits. Cette déqualification du quartier semble être reliée à la conjonction de plusieurs facteurs liés au positionnement à l'intérieur de l'agglomération et au sein d'une région périphérique. Ainsi, Kénogami s'est retrouvée isolée par la construction récente de l'autoroute 70 tout en vivant les contrecoups de la crise forestière.

L'objectif de cette recherche est de réaliser un portrait historique, urbanistique et socioéconomique de ce territoire en mal de développement de même que de comparer les stratégies de revitalisation actuellement en cours (stratégies commerciale, patrimoniale et communautaire). Nous tenterons également de vérifier dans quelle mesure les stratégies de revitalisation rejoignent l'approche de la revitalisation urbaine intégrée. Cette approche actuellement en vogue se veut globale, participative et multisectorielle ${ }^{4}$. Elle tranche avec les démarches du passé fondées essentiellement sur les infrastructures urbaines et le mixte commercial.

Contrairement à sa voisine Arvida, Kénogami n'a pas été en mesure d'utiliser sa forme urbaine particulière pour se mettre en valeur et attirer résidants et industries.

Sur le plan méthodologique, quatre sources et méthodes seront utilisées: les sources bibliographiques et historiques sur Kénogami, les visites de terrains à des fins photo-cartographiques, les données socioéconomiques des recensements canadiens de 1981 à 2006, de même que les matériaux et expertises développés par la défunte Société d'intervention urbaine de Chicoutimi-Jonquière (SIU) en 
matière de mobilisation communautaire. En ce qui concerne l'ordre des idées, nous traiterons d'abord de la naissance et de l'évolution de Kénogami. Par la suite, nous analyserons les récentes stratégies et interventions de développement mises de l'avant par divers acteurs pour relancer la dynamique socioéconomique du quartier.

\section{NAISSANCE ET ÉVOLUTION DE KÉNOGAMI}

\subsection{Occupation et organisation du territoire}

L'histoire de Kénogami se confond avec celle de Jonquière, l'ancienne ville voisine dont le tissu urbain est contigu au sien. C'est en 1866 que la municipalité du canton de Jonquière voit le jour. Auparavant, le territoire du canton de Jonquière était rattaché à la municipalité de Chicoutimi. Entre 1851 et 1871 , la population du canton de
Jonquière est passée de 82 à 1290 personnes, ce qui justifiait la création d'une municipalité selon les critères de l'époque ${ }^{5}$. En 1897, la partie sud du canton de Jonquière est détachée pour créer la nouvelle municipalité de canton de Kénogami. Avec la construction d'une usine de papier, la municipalité du village de Kénogami est créée en 1912 et celle-ci devient une ville, au sens légal du terme, en 1920 (voir la carte 1).

\section{Carte 1 : Kénogami : évolution du territoire municipal}

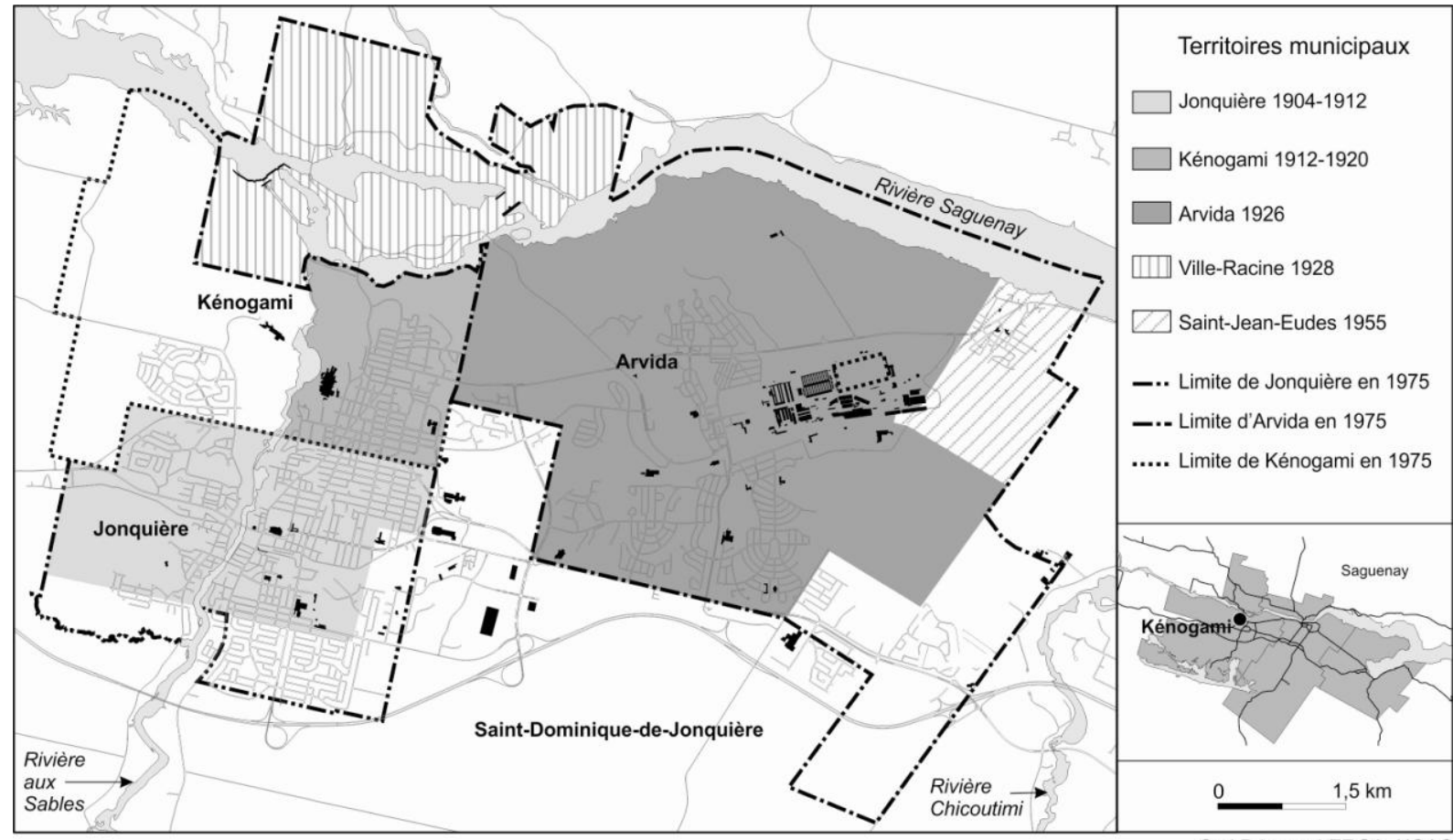

Carl Brisson, LERGA, UQAC

Kénogami est une ville mono-industrielle construite dans le but d'accueillir les activités de la Price Brothers cie. et de loger ses employés. Il s'agit donc d'une ville planifiée arborant un plan urbain en damier où les cadres et les professionnels (souvent anglophones) habitent un quartier distinct de celui des ouvriers « canadiensfrançais ».
Le Canton de Jonquière a connu un parcours de développement assez classique : arpentage des terres, peuplement agricole progressif et constitution d'un noyau urbain autour des services religieux et commerciaux implantés. Dans le cas de Kénogami, et incidemment d'Arvida, la collectivité locale doit sa naissance au choix fait par des industriels d'ériger une usine en ce lieu. Ainsi, Kénogami est une ville mono-industrielle construite dans le but d'accueillir les activités de 
la Price Brothers cie et de loger ses employés. Il s'agit donc d'une ville planifiée arborant un plan urbain en damier où les cadres et les professionnels (souvent anglophones) habitent un quartier distinct de celui des ouvriers «canadiens-français ». Malgré l'existence d'une corporation municipale, la ville et ses infrastructures ont été la propriété de l'entreprise jusqu'à ce que celle-ci se désengage progressivement de ses biens, entre 1922 et 1957 (systèmes d'égout et d'eau potable, rues et habitations) ${ }^{6}$.

Dans les premières décennies de son existence, la population de Kénogami est en constante progression au même titre que celle des municipalités voisines (voir le tableau 1). Bien que Jonquière ait une vocation industrielle plus ténue que Kénogami et Arvida, elle est la plus populeuse avec un total de 13769 citoyens en 1941. Déjà, à cette époque, Jonquière affirme sa vocation résidentielle. La période de l'aprèsguerre verra ces municipalités connaître un accroissement important de leur population et une augmentation sensible des conditions de vie des résidants. À l'aube des années 1960, l'utilisation de plus en plus massive de l'automobile, l'accès à des résidences unifamiliales, l'arrivée des grands «magasins à rayons » entraînent une forte consommation d'espace qui fera éclater les limites du périmètre urbain. L'exiguïté des territoires municipaux de Kénogami et de Jonquière rendent pratiquement impossible la venue de développements résidentiels et industriels majeurs à moins de modifier les frontières municipales. Ce qui se produira à diverses reprises.

Tableau 1 : Variation de la population entre 1901 et 2001

\begin{tabular}{|c|c|c|c|c|c|}
\hline & $\begin{array}{c}\text { St-Dominique- } \\
\text { de-Jonquière }\end{array}$ & Arvida & Jonquière & Kénogami & $\begin{array}{c}\text { Jonq. regroupé } \\
\text { ou équivalent }\end{array}$ \\
\hline $\mathbf{1 9 0 1}$ & 1630 & - & - & - & 1630 \\
\hline $\mathbf{1 9 1 1}$ & $1078(-33,9 \%)$ & - & 2354 & - & $3432(110,6 \%)$ \\
\hline $\mathbf{1 9 2 1}$ & $1213(12,5 \%)$ & - & $4851(106,1 \%)$ & 2557 & $8621(151,2 \%)$ \\
\hline $\mathbf{1 9 3 1}$ & $1350(11,3 \%)$ & 1790 & $9448(94,8 \%)$ & $4500(76,0 \%)$ & $17193(99,4 \%)$ \\
\hline $\mathbf{1 9 4 1}$ & $1685(24,8 \%)$ & $4581(155,9 \%)$ & $13769(45,7 \%)$ & $6579(46,2 \%)$ & $26786(55,8 \%)$ \\
\hline $\mathbf{1 9 5 1}$ & $2093(24,2 \%)$ & $11078(141,8 \%)$ & $21618(57,0 \%)$ & $9895(50,4 \%)$ & $44684(66,8 \%)$ \\
\hline $\mathbf{1 9 6 1}$ & $3939(88,2 \%)$ & $14460(30,5 \%)$ & $28588(32,2 \%)$ & $11816(19,4 \%)$ & $61676(38,0 \%)$ \\
\hline $\mathbf{1 9 7 1}$ & $4591(16,7 \%)$ & $18448(27,6 \%)$ & $28430(-0,6 \%)$ & $10970(-7,2 \%)$ & $62444(1,2 \%)$ \\
\hline $\mathbf{1 9 8 1}$ & - & - & - & - & $60354(-3,3 \%)$ \\
\hline $\mathbf{1 9 9 1}$ & - & - & - & - & $57933(-4,0 \%)$ \\
\hline $\mathbf{2 0 0 1}$ & - & - & - & - & $54842(-5,3 \%)$ \\
\hline
\end{tabular}

En 1953, la cité de Kénogami annexe une partie de la municipalité de paroisse de SaintDominique-de-Jonquière. Ce territoire situé à l'ouest de la Rivière-aux-Sables, que l'on nomme maintenant Jonquière-Nord, comprend une partie du rang Saint-André et du rang SaintThomas. Cet ajout de territoire permet à la cité de Kénogami de tripler sa superficie. Les autorités municipales peuvent dorénavant envisager la planification de nouveaux développements. Cependant, l'aménagement du secteur Kénogami-Ouest nécessite la construction d'un pont sur la Rivière-aux-Sables afin de le relier plus directement au Vieux-Kénogami. Un premier projet est annoncé en 1952 mais celui-ci ne verra pas le jour en raison de l'opposition de la compagnie Price Brothers ${ }^{6}$.

Afin de mieux contrôler l'expansion urbaine sur son territoire, la municipalité de Kénogami adopte son premier Plan directeur d'urbanisme en 1961. Parmi les projets contenus dans le plan, on retrouve l'augmentation de la qualité de l'habitation, l'amélioration des facilités de stationnement au centre-ville, la planification et le développement d'un nouveau secteur résidentiel à l'ouest de la Rivière-aux-Sables (Kénogami-Ouest) ainsi que l'aménagement d'un parc industriel. 
Afin de relier les deux secteurs de Kénogami, un référendum est tenu afin de choisir parmi trois scénarios celui qui est le plus favorable à la construction d'un pont. C'est le tracé de la rue Nelson qui est favorisé au détriment de la rue Du-Roi-Georges. La construction débuta en juillet 1963 et le pont Nelson est ouvert à la circulation en novembre 1965. La présence du pont qui permet le passage de la route régionale désenclave grandement Kénogami. Au début des années 1970, les premières habitations à loyer modique (HLM) sont construites sur la nouvelle rue des Buissons, tout près de l'intersection du rang Saint-André et de la rue Nelson. Par la suite, un nouveau secteur résidentiel prend forme au même endroit (voir la carte 3). Celui-ci se distingue fortement de la partie ancienne de Kénogami par son réseau de rues de type curvilinéaire et son habitat de style «bungalow». Paradoxalement, le déclin démographique de la municipalité commence à se faire sentir. Ainsi, en 1970-1971, il n'y a eu que 31 nouvelles constructions unifamiliales et 17 constructions multifamiliales ${ }^{7}$.

\section{Carte 2 : Kénogami : historique du développement urbain}

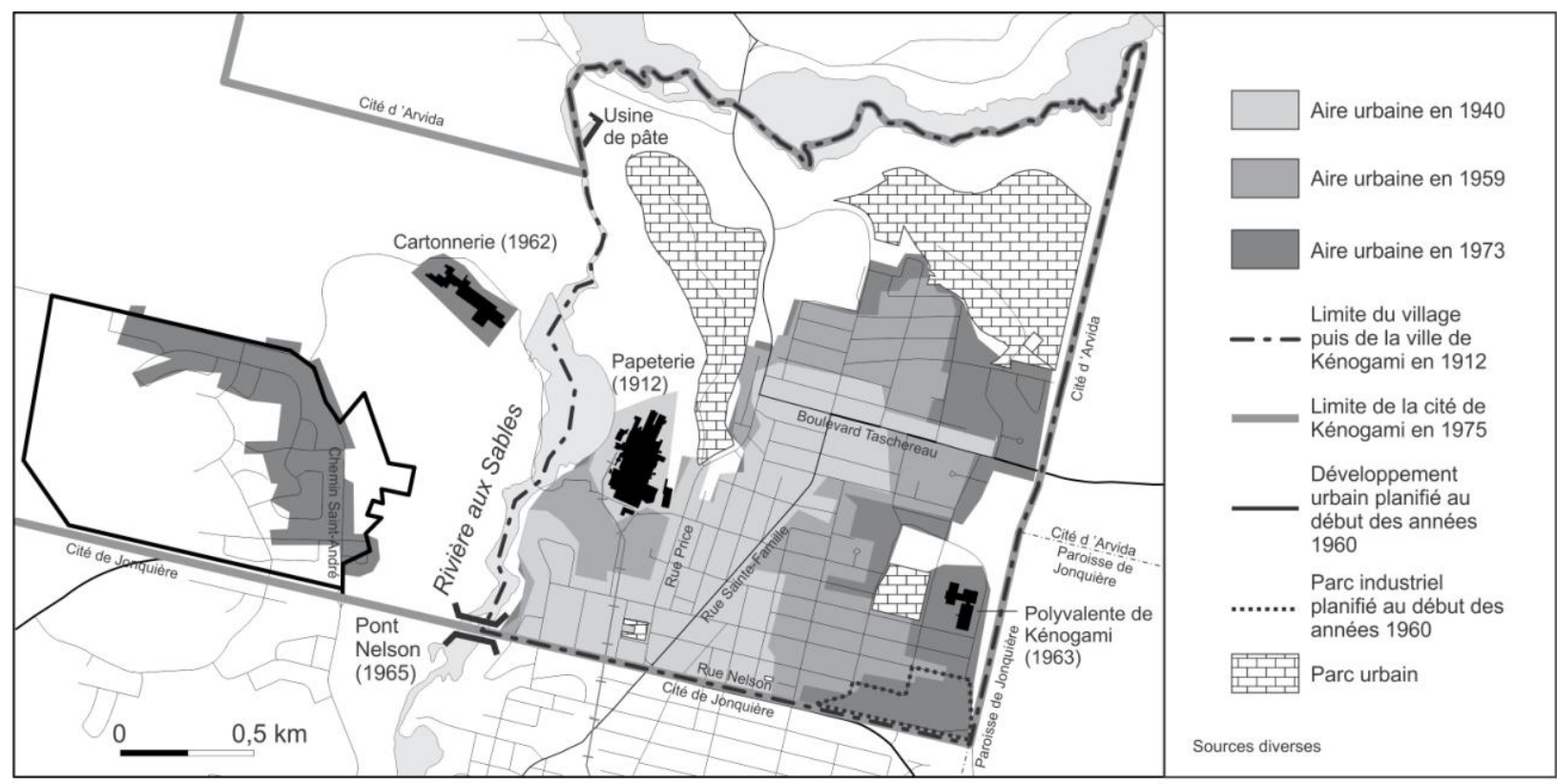

Carl Brisson, LERGA, UQAC, 2010

1.2 Regroupements administratifs et politiques d'urbanisme

\section{En juin 1972, le ministère des Affaires municipales annonce qu'en vertu de la loi favorisant le regroupement des municipalités, une unité de regroupement comprenant Jonquière, Kénogami, d'Arvida et la paroisse de Saint- Dominique-de-Jonquière est établie.}

Dans les années 1960, la zone urbaine de Jonquière rejoint celle de Kénogami et forme un espace urbain continu de plusieurs kilomètres aux abords de la Rivière-aux-Sables. Cet espace compte alors près de 42000 habitants $^{6}$. Ainsi, l'idée d'un regroupement municipal entre les deux villes sœurs fait son chemin. La richesse foncière est l'un des enjeux du débat. En effet, grâce aux industries qui les ont créées, les villes de Kénogami et d'Arvida sont bien nanties financièrement mais il n'en est pas de même pour Jonquière. En juin 1972, le ministère des Affaires municipales annonce qu'en vertu de la loi favorisant le regroupement des municipalités, une unité de regroupement comprenant Jonquière, Kénogami, Arvida et la paroisse de Saint-Dominique-de-Jonquière est établie ${ }^{7}$. La nouvelle Ville de Jonquière est créée en $1975^{8}$. 
L'année suivante, la Ville de Chicoutimi, qui est située tout juste à l'Est, fait l'objet d'un regroupement qui lui donne une taille semblable à celle de Jonquière.

En 1976, la Ville regroupée de Jonquière se dote d'un premier Plan d'urbanisme. Les locaux de l'ancienne mairie d'Arvida ont été choisis à titre de nouvel hôtel de ville. Le concept d'organisation de l'espace s'articule autour de l'accessibilité. Le réseau routier constitue l'élément central indispensable à l'intégration des noyaux anciens du tissu urbain et à l'intégration des nouveaux équipements collectifs. En matière commerciale, le concept d'aménagement prévoit le maintien des trois anciens centres-villes de Jonquière, d'Arvida et de Kénogami. Cependant, une artère commerciale périphérique est consolidée sur la route 170 et celle-ci se prolonge sur le boulevard Harvey. Le déplacement du centre de gravité vers le sud de la ville trouvera son point culminant en 2004 avec l'ouverture de l'autoroute 70 qui ceinture la région métropolitaine de Saguenay.

En 1982, la ville de Jonquière adopte un deuxième Plan d'urbanisme. Les transports demeurent une priorité, notamment à travers l'implantation du boulevard de la Centrale (devenu boul. René-Lévesque) qui sera considéré comme étant l'épine dorsale des déplacements nord-sud. Les développements domiciliaires dans ce secteur ont clairement pour but de relier spatialement les aires urbanisées d'Arvida et de Kénogami ${ }^{9}$. De plus, la loi sur la protection $\mathrm{du}$ territoire agricole, nouvellement en vigueur, oblige un certain resserrement de la trame urbaine, comme partout au Québec. Eu égard aux commerces et services publics, le plan de 1982 prône une concentration sur la rue SaintDominique et met en branle un Programme particulier d'urbanisme. Ce plan d'action réalisé par la firme conseil Daniel Arbour et associés est adopté en 1985. En 2005, une étude sur les artères commerciales traditionnelles de la Ville de Saguenay nous rappelle que les problèmes de développement des rues commerciales traditionnelles sont récurrents ${ }^{10}$, notamment sur la rue Sainte-Famille à Kénogami (voir la photo 1).

En 1991, la Ville de Jonquière adopte son troisième Plan d'urbanisme. Ce nouveau document de planification territoriale est devenu nécessaire compte tenu des transformations sur le territoire ainsi que par l'adoption d'un Schéma d'aménagement par la municipalité régionale de comté (MRC) du Fjord-duSaguenay. On constate le déclin de la population de Jonquière. Dans le domaine industriel, de nombreuses pertes d'emplois industriels sont à prévoir en raison de la modernisation des équipements, en particulier chez Alcan (devenu RTA en 2009). Le Plan d'urbanisme reconnaît également la faiblesse de l'offre touristique jonquiéroise même si de nouvelles infrastructures ont vu le jour, tel le site de plein-air CÉPAL. En fait, tous les domaines de l'activité socioéconomique de la ville nécessitent des interventions afin de contrer la stagnation et la morosité qui semblent s'implanter.

À la fin des années 1990, le gouvernement du Québec était convaincu de la nécessité d'apporter des changements au système municipal. En 2000 et 2001, le gouvernement adopta une série de lois et de décrets qui permettront de renforcer les agglomérations urbaines tout en diminuant le nombre de municipalités. C'est donc dans ce contexte que la Ville de Saguenay fut créée à la suite de l'adoption par le gouvernement québécois du décret 871-2001, le 26 juin 2001. La nouvelle Ville de Saguenay, laquelle prend vie le 18 février 2002, regroupe les anciennes municipalités de Chicoutimi, Jonquière, La Baie, Laterrière, de même que Shipshaw, Lac-Kénogami et une partie de Canton Tremblay. Depuis ce temps, un certain nombre de dossiers relatifs au fonctionnement de la ville ont été harmonisés. Néanmoins, la mise en place d'un nouveau Plan d'urbanisme tarde alors que le Schéma d'aménagement et de développement est sur le point d'être adopté. Ainsi, en ce qui concerne l'arrondissement de Jonquière, c'est le Plan d'urbanisme de l'ex-ville de Jonquière qui est toujours en vigueur, au moment d'écrire ce texte.

La nouvelle Ville de Saguenay, laquelle prend vie le 18 février 2002 regroupe les anciennes municipalités de Chicoutimi, Jonquière, La Baie, Laterrière, de même que Shipshaw, Lac-Kénogami et une partie de CantonTremblay. 
Photo 1 : La rue Sainte-Famille à Kénogami (source : Google Earth, 2010)

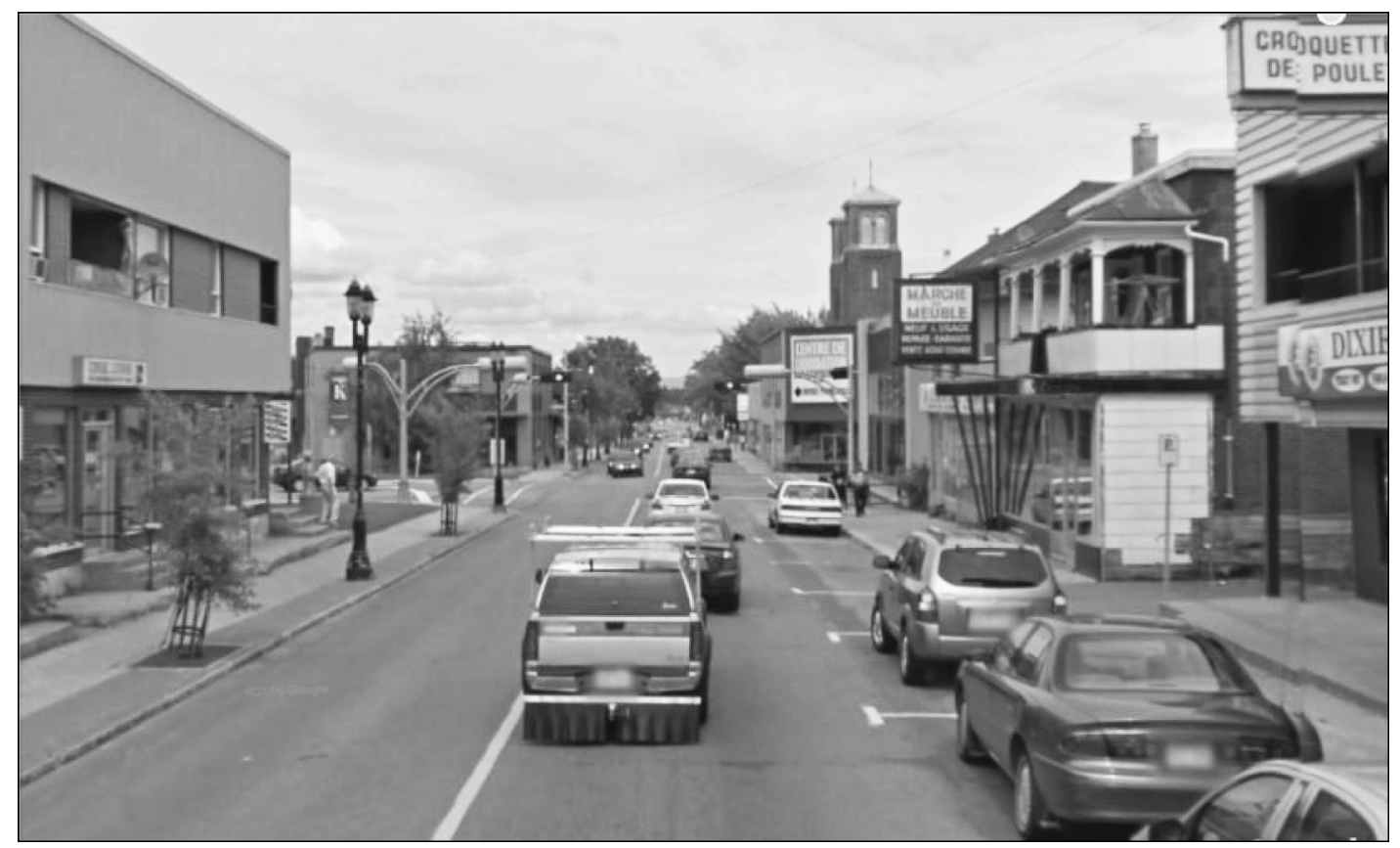

1.3 Le déclin socioéconomique progressif de Kénogami

En quarante ans, les emplois industriels ont donc diminué de $75 \%$ dans l'arrondissement de Jonquière. En ce qui concerne la démographie, on remarque également une décroissance constante de la population.

Dès la première moitié du $X X X^{\mathrm{e}}$ siècle, l'ensemble formé de Jonquière-Arvida-Kénogami (JAK) se hisse au premier rang des espaces industriels de la région alors qu'il accueille une pulperie (Jonquière), une papeterie et une cartonnerie (Kénogami), une aluminerie, une usine chimique et des grandes centrales hydroélectriques (Arvida). Mentionnons que la pulperie de Jonquière a fermé ses portes en 1963. En termes d'emplois, ce sont des milliers de travailleurs qui œuvrent dans les installations industrielles de l'agglomération JAK. Par exemple en 1970, l'usine de pâtes et papiers de Kénogami emploie 1000 personnes alors que la cartonnerie fait travailler 342 personnes. À Arvida, les installations de l'Alcan occupent 6452 personnes en 1970. Ces trois complexes embauchent donc près de 8000 personnes.
Depuis ce temps, la situation de l'emploi industriel dans l'agglomération connaît une diminution constante. En 2009, on ne retrouvait que 389 emplois à l'usine de pâtes et papiers de Kénogami, 168 emplois à la cartonnerie et 1975 emplois aux installations d'Alcan à Arvida ${ }^{11}$. En quarante ans, les emplois industriels ont donc diminué de $75 \%$ dans l'arrondissement de Jonquière. En ce qui concerne la démographie, on remarque également une décroissance constante de la population. Cette tendance affecte l'ensemble de la région administrative $\mathrm{du}$ Saguenay_Lac-Saint-Jean et la Ville de Saguenay. Toutefois, cette diminution a touché l'arrondissement de Jonquière, et donc le quartier de Kénogami, plus tôt, soit - 3,3\% entre 1971 et $1981,-4 \%$ entre 1981 et $1991,-5,3 \%$ entre 1991 et 2001. Enfin, depuis le regroupement de 2002, la population de Jonquière continue de décroître. Entre 2001 et 2006, le bilan des pertes s'établit à 1393 personnes, soit une baisse de $2,5 \%$ de la population totale.

Enfin, depuis le regroupement de 2002, la population de Jonquière continue à décroître. Entre 2001 et 2006, le bilan des pertes s'établit à 1393 personnes, soit une baisse de $2,5 \%$ de la population totale. 
Le quartier de Kénogami, lequel compte aujourd'hui environ 5500 habitants, affiche plusieurs caractéristiques des milieux défavorisés, notamment un revenu moyen par ménage inférieur à celui de l'arrondissement et de la région métropolitaine de recensement et ce, même, parmi les quartiers dévitalisés de Saguenay (voir le tableau $2)$. On observe également une détérioration du milieu physique et des équipements, malgré le réaménagement récent du Parc Price aux coûts de $\$ 2,5$ millions (voir la photo. 2), et l'abandon de plusieurs églises monumentales. La population résidante ne semble plus battre au diapason de l'usine, les travailleurs moins nombreux mais bien rémunérés demeurant en majorité hors du centre-ville ou du quartier. Certaines institutions comme la polyvalente de Kénogami sont actives et bien réputées. Néanmoins, cette vitalité est liée à des programmes spéciaux qui attirent les jeunes d'autres quartiers ou d'autres arrondissements.

Tableau 2 : Kénogami dans le contexte de la RMR de Saguenay ${ }^{12}$

\begin{tabular}{|l|c|c|c|c|c|c|c|c|}
\hline & \multicolumn{2}{|c|}{ Kénogami } & \multicolumn{2}{c|}{ Arr. de Jonquière } & \multicolumn{2}{c|}{ Ville de Saguenay } & \multicolumn{2}{c|}{ Québec } \\
\hline & $\mathbf{1 9 9 6}$ & $\mathbf{2 0 0 6}$ & $\mathbf{1 9 9 6}$ & $\mathbf{2 0 0 6}$ & $\mathbf{1 9 9 6}$ & $\mathbf{2 0 0 6}$ & $\mathbf{1 9 9 6}$ & $\mathbf{2 0 0 6}$ \\
\hline Population totale & 9238 & 6054 & 60878 & 58474 & 153476 & 141680 & 7138795 & 7546131 \\
\hline Revenu moyen des ménages & 37593 & 43724 & 40015 & 54292 & 40968 & 55654 & 42229 & 58954 \\
\hline Taux d'activité & 54,4 & 52,8 & 56,0 & 58,6 & 57,8 & 59,9 & 62,3 & 64,9 \\
\hline Taux d'emploi & 45,1 & 47,0 & 48,1 & 53,1 & 50,2 & 54,7 & 55,0 & 60,4 \\
\hline Taux de chômage & 17,1 & 11,0 & 14,2 & 9,3 & 13,1 & 8,7 & 11,8 & 7,0 \\
\hline Taux de familles monoparentales & 16,8 & 20,0 & 15,4 & 15,7 & 15,0 & 15,1 & 15,8 & 16,6 \\
\hline \% de personnes vivant seules & 9,2 & 17,4 & 8,7 & 12,9 & 8,6 & 12,7 & 10,9 & 13,3 \\
\hline \% de personnes à faible revenu & ND & 23,4 & 23,0 & 15,4 & 20,8 & 14,5 & 23,4 & 17,2 \\
\hline \% sans diplôme d'études secondaires & 32,3 & 25,5 & 31,4 & 22,2 & 31,3 & 22,1 & 34,9 & 25,0 \\
\hline
\end{tabular}

Photo 2 : Le parc Price à Kénogami qui a été rouvert en 2010 (source : M. Simard, 2010)

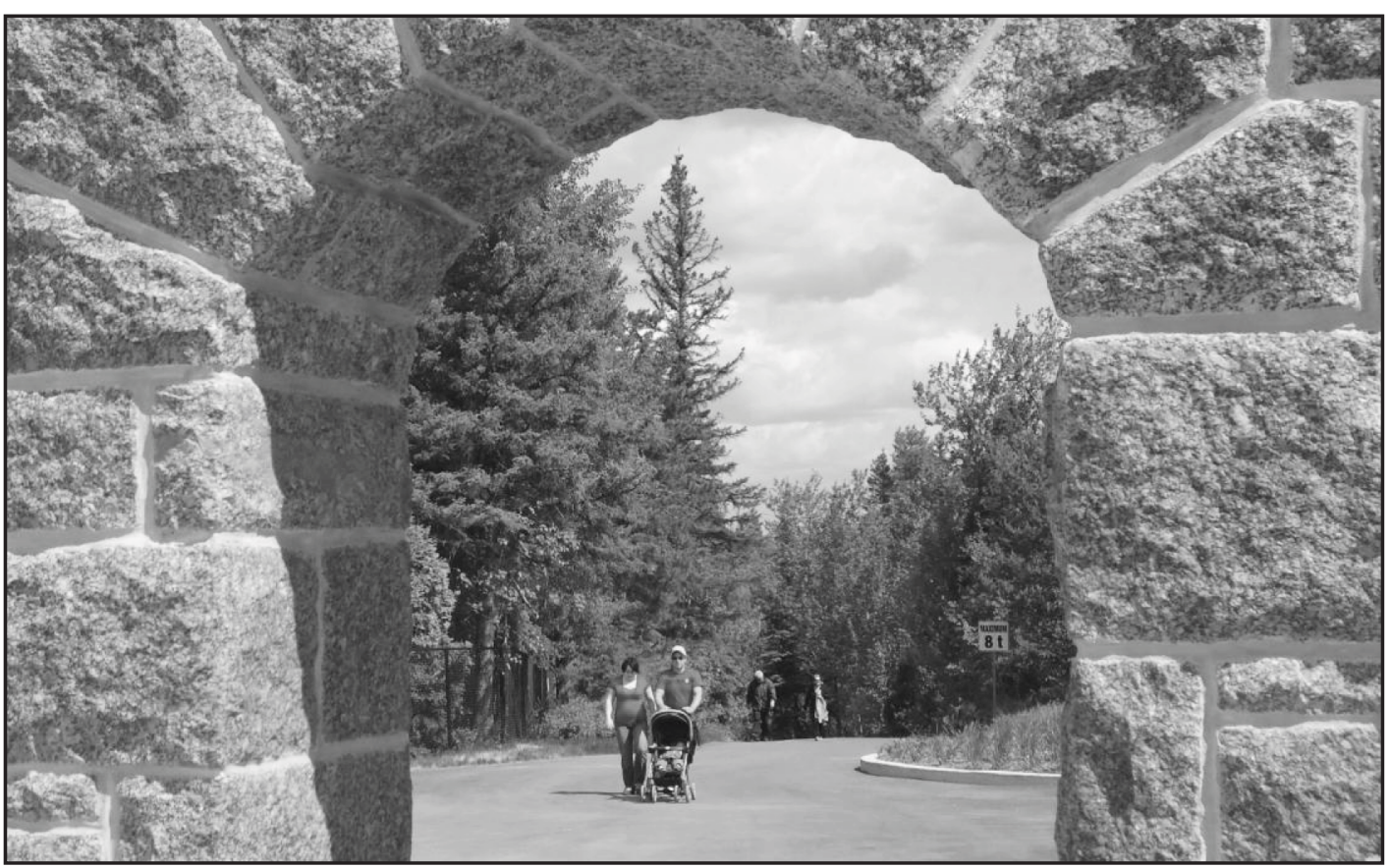




\section{LES STRATÉGIES DE REVITALISATION}

Le quartier de Kénogami est un espace défavorisé dans le contexte actuel. La carte des aires sociales du groupe ÉCOBES a démontré en 1997 la position relative désavantageuse de Kénogami à l'intérieur de l'agglomération de Saguenay ${ }^{13}$. De plus, la Société d'intervention urbaine de Saguenay (SIU), un organisme de développement local financé par le gouvernement fédéral entre 1992 et 2010, intégrait Kénogami dans sa liste des quartiers en difficulté des arrondissements de Chicoutimi et de Jonquière. Il est aussi opportun d'analyser les différentes stratégies et interventions entreprises au cours de la dernière décennie en vue de requalifier le milieu et d'améliorer les conditions de vie des résidants.

\subsection{La mise en valeur du patrimoine}

À titre d'ancienne ville planifiée par des industriels, Kénogami présente une forme urbaine et des paysages particuliers. Cette originalité se

Anglais (voir la photo. 3), soit la partie ouest du quartier où habitaient les cadres anglophones travaillant à l'usine. On y retrouve des bâtiments de style néo-tudor. Cette vocation historique est mise en valeur depuis une douzaine d'années par le Centre historique Sir-William-Price. Cette institution muséale reconnue par le ministère de la Culture et des Communications du Québec offre des visites guidées de Kénogami, au cours des mois d'été. Le Centre Sir-William-Price est issu d'une initiative locale et il est géré par l'organisme Patrimoine Saguenay, anciennement nommé Patrimoine Jonquière. révèle principalement dans le secteur des

Photo 3 : Le secteur « des Anglais » à Kénogami (source : M. Simard, 2010)

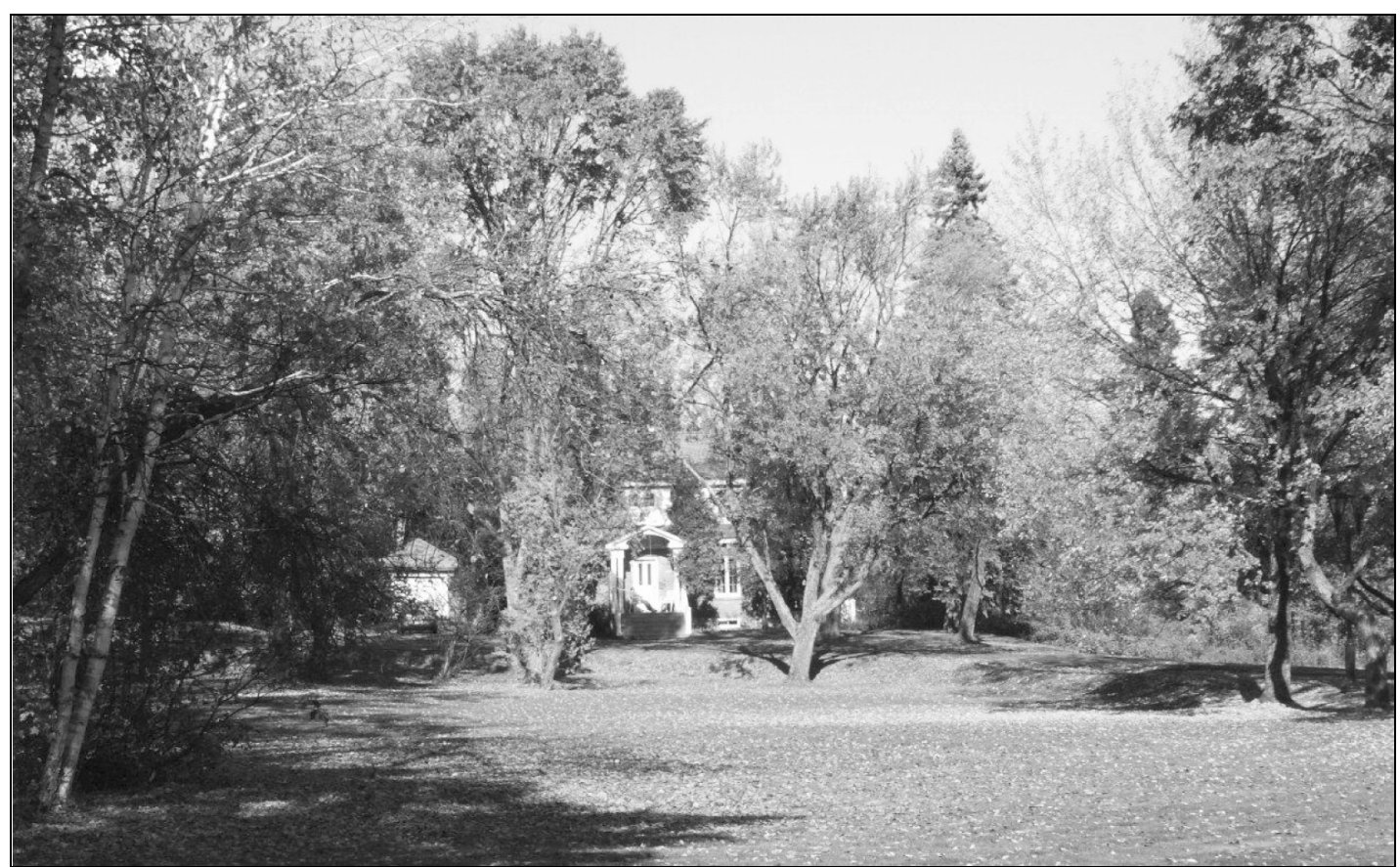




\section{La corporation Patrimoine Jonquière a été fondée en 1987 par un petit groupe de citoyens de la ville de Jonquière, principalement pour sauvegarder une petite chapelle anglicane qui se trouvait à Kénogami et qui était menacée de démolition.}

La corporation Patrimoine Jonquière a été fondée en 1987 par un petit groupe de citoyens de la Ville de Jonquière, principalement pour sauvegarder une petite chapelle anglicane qui se trouvait à Kénogami et qui était menacée de démolition. Le mandat de cet organisme à but non lucratif « est de protéger, de préserver et de valoriser le patrimoine bâti, les sites et œuvres historiques, culturels, artistiques, et d'en faire la promotion $^{14} »$. Le premier dossier sur lequel s'est penchée la corporation Patrimoine Jonquière est donc celui de la sauvegarde de la chapelle anglicane.

La chapelle a été déménagée dans le parc Ball dans le secteur Kénogami de l'arrondissement de Jonquière et restaurée, notamment grâce à l'aide financière de la famille Price. Dès 1988, le bâtiment restauré a été inauguré comme musée sur l'histoire de la compagnie Price. Depuis ce temps, le petit musée est devenu le Centre d'histoire Sir-William-Price. Il a pour mission de faire connaître « la dynastie d'affaires de la famille Price et l'histoire de la colonisation du Saguenay ainsi que celle de l'industrie forestière et des pâtes et papiers ».

Patrimoine Jonquière est devenue Patrimoine Saguenay en 2008 et il œuvre aujourd'hui partout sur le territoire de la ville regroupée de Saguenay ${ }^{15}$. Au cours de la dernière décennie, des projets ont été réalisés à Jonquière (rénovation de l'ancienne résidence des Frères du Sacré-Cœur) et à Arvida (reconversion en habitations de l'école Saint-Mathias). Les interventions de l'organisme patrimonial ont des retombées positives à Kénogami, bien que son action se situe davantage dans une vision sectorielle que territoriale du développement. Même si le Centre d'histoire Sir-William-Price crée peu d'emplois, il contribue à la réhabilitation du domaine bâti ainsi qu'à la revalorisation symbolique du quartier. Paradoxalement, Patrimoine Saguenay ne jouit pas du support constant de la municipalité, laquelle se déclare en faveur de la requalification du secteur. Ainsi, les difficultés rencontrées par l'organisme sont la reconnaissance de son rôle dans la préservation du patrimoine bâti et la fragilité des montages financiers indispensables à la réalisation des projets.

\subsection{Le développement commercial}

Le centre-ville de Jonquière constitue le deuxième pôle d'emploi en importance à Saguenay. Le centre d'affaires traditionnel de Kénogami est beaucoup plus modeste (voir le tableau 3). On y dénombre seulement 564 emplois en 2006, surtout des emplois dans le commerce $(67,7 \%)$. De plus, le commerce y est relativement peu diversifié et les établissements en activité occupent des créneaux que l'on pourrait qualifier de «bas de gamme». Par exemple, on retrouve un centre de liquidation de meubles, des friperies et de la restauration rapide hors bannière de style « casse-croûte ».

Les centres-villes traditionnels de Saguenay éprouvent tous des difficultés, mais Kénogami est l'un de ceux qui souffrent le plus d'anémie. Dans ce contexte, la revitalisation du centre-ville de Kénogami occupe une place importante dans le débat public. Par ailleurs, la population du quartier et les commerçants sont sensibles aux interventions municipales récentes qui paraissent concentrées du côté de Jonquière (rénovation du Palais des sports, érection d'un bureau touristique et projet de nouvelle bibliothèque d'arrondissement).

Les centres-villes traditionnels de

Saguenay éprouvent tous des difficultés, mais Kénogami est l'un de ceux qui souffrent le plus d'anémie. Dans ce contexte, la revitalisation du centre-ville de Kénogami occupe une place importante dans le débat public. 
Tableau 3 : Nombre d'emplois par secteur d'activité dans les centres-villes en 2007

\begin{tabular}{|l|c|c|l|}
\hline Centre-ville & Jonquière & Arvida & Kénogami \\
\hline Commercial & $1876(51,1 \%)$ & $616(33,9 \%)$ & $382(67,7 \%)$ \\
\hline Services aux entreprises & $483(13,1 \%)$ & $548(30,2 \%)$ & $102(18,1 \%)$ \\
\hline Industriel & $102(2,8 \%)$ & $56(3,1 \%)$ & $1(0,2 \%)$ \\
\hline Services publics & $1213(33,0 \%)$ & $595(32,8 \%)$ & $79(14,0 \%)$ \\
\hline Total & $3674(100 \%)$ & $1815(100 \%)$ & $564(100 \%)$ \\
\hline
\end{tabular}

La question de la vitalité commerciale est évidemment au cœur de la problématique de la revitalisation des centres-villes à Saguenay. Les commerces de Kénogami se situent principalement sur la rue Sainte-Famille et sur la rue $\mathrm{Du}$ Roi-Georges. Le rapport de la firme Paquin et associés sur le commerce à Saguenay, rapport qui a été commandé par Promotion Saguenay en 2005, confirme la faible popularité de Kénogami comme destination commerciale. En effet, ce secteur qui rassemble 70 locaux commerciaux pour une superficie commerciale totale de 185301 pieds carré est décrit de manière peu élogieuse dans le rapport :

«[malgré] peu de locaux vacants [et] une accessibilité régionale assez bonne, l'image générale des commerces est souvent dégradée. On y trouve plusieurs bazars et magasins de marchandises usagées. Les commerces ont souvent des façades peu attirantes et leur affichage est vieillissant ${ }^{10} »$.

Récemment, la Ville de Saguenay, par le biais de l'organisme Promotion Saguenay, a débuté la mise en œuvre d'un plan d'action dans le cadre de la revitalisation des artères commerciales traditionnelles. À Kénogami, les interventions publiques consistent à repaver certains tronçons de rue et à remodeler les trottoirs et le mobilier urbain, principalement sur les rues SainteFamille et Du-Roi-George. De plus, on vise à favoriser l'animation urbaine en fermant certaines voies publiques à la circulation automobile durant la période d'été. Finalement, les autorités municipales ont procédé à des démolitions d'édifices et à des remembrements fonciers en vue d'éventuels projets immobiliers de nature commerciale. Ces actions comprennent aussi la réfection du parc Price (voir la photo 2). En 2007, Promotion Saguenay débute un processus de concertation avec les commerçants locaux. Ainsi, l'Association des gens d'affaires devient la Corporation de vitalisation de Kénogami, soit un organisme géré par un Conseil d'administration de 12 membres issus du milieu.

\subsection{Le développement communautaire}

Le secteur de Kénogami a été l'objet aussi de plusieurs interventions sur le plan communautaire. La Société d'intervention urbaine ${ }^{17}$ est un organisme qui était, jusqu'à tout récemment, voué au développement économique communautaire et à la revitalisation sociale et économique des arrondissements de Chicoutimi et de Jonquière. Elle a réalisé de nombreuses interventions en vue de stimuler les relations sociales et d'œuvrer à la redynamisation de Kénogami dans les sphères communautaire, sociale et économique. Identifiée dès 2002 comme étant un secteur en dévitalisation, la Société d'intervention urbaine est intervenue fréquemment dans Kénogami. Parmi les projets réalisés en partenariat avec plusieurs organismes du milieu, on peut mentionner le soutien à la relance de l'Association des gens d'affaires de Kénogami. En effet, plusieurs partenaires locaux ont travaillé à réanimer cette association qui joue maintenant un rôle important comme acteur du développement local. 
Ainsi, entre 2002 et 2004, les gens d'affaires du secteur accompagnés par des représentants du Centre local de développement, de la Société d'intervention urbaine et de la Corporation de sauvegarde du patrimoine de Jonquière ont développé un plan d'action en vue d'améliorer l'aménagement urbain du secteur commercial de Kénogami. Notamment, des oriflammes aux couleurs de Kénogami ont été installées dans l'optique de contribuer au sentiment d'appartenance des gens d'affaires et de la population du quartier. Plusieurs autres initiatives ont été mises de l'avant comme l'instauration d'une fête de quartier annuelle, des ventes trottoirs, des concerts du midi, la promotion de l'achat local, l'amélioration des espaces verts, dont le Parc Ball qui est au cœur du quartier.

\section{Entre 2002 et 2004, les gens d'affaires du secteur accompagnés par des représentants du Centre local de développement, de la Société d'intervention urbaine, et de la Corporation de sauvegarde du patrimoine de Jonquière ont développé un plan d'action en vue d'améliorer l'aménagement urbain du secteur commercial de Kénogami.}

En avril 2005, la Société d'intervention urbaine Chicoutimi-Jonquière tenta d'aller encore plus loin en organisant une assemblée de quartier nommée pour l'occasion La levée d'idées. Cette assemblée préparée par un comité composé de partenaires sociaux et économiques avait pour objectif de «contribuer à faire de Kénogami un milieu où il fait bon vivre et où tous (citoyens et organisations) y trouvent leur compte ${ }^{18} »$. Il s'agissait également de «sensibiliser les citoyens et organisations (publiques et privées) du secteur Kénogami sur leur capacité à contribuer collectivement au devenir de leur milieu ».

À la suite de cette assemblée publique, la Société d'intervention urbaine a essayé de créer un comité de suivi composé de citoyens afin de mettre en œuvre les actions prioritaires proposées lors de l'assemblée. Les intérêts divergents, souvent liés à la politique locale, et le manque d'engagement de certains membres ont rendu difficile, voire impossible, le maintien de ce comité.

\section{Plusieurs autres initiatives ont été mises de l'avant comme l'instauration d'une fête de quartier annuelle, des ventes trottoirs, des concerts du midi, la promotion de l'achat local, \\ l'amélioration des espaces verts, dont le Parc Ball qui est au cour du quartier.}

Comme cela a déjà été mentionné dans un article antérieur $^{19}$, même si l'expérience d'assemblées publiques de quartier apparait somme toute assez positive, l'idée de réunir divers types d'acteurs sociaux, économiques, politiques, de même que des citoyens, dans un même lieu s'avère assez difficile à mettre en œuvre.

À Kénogami, les gens d'affaires ont participé en grand nombre à l'évènement, mais la population du secteur est demeurée en grande partie à l'écart du processus. La situation et la dynamique sociale du secteur de Kénogami semblent particulières, puisque les simples citoyens ont davantage participé dans d'autres quartiers de Saguenay. Les commerçants qui sont regroupés au sein d'une association spécifique demeurent actifs à l'intérieur d'un organisme utile mais qui parle inévitablement d'un point de vue sectoriel.
À Kénogami, les gens d'affaires ont participé en grand nombre à
l'évènement, mais la population du secteur est demeurée en grande partie à
l'écart du processus. La situation et la dynamique sociale du secteur de Kénogami semblent particulières, puisque les simples citoyens ont davantage participé dans d'autres quartiers de Saguenay. 


\section{CONCLUSION}

\section{Le déclin et la paupérisation du quartier Kénogami constituent malheureusement des faits avérés en 2011. Les indicateurs de toutes sortes confirment aisément ce constat de même que les visites de terrain.}

Le déclin et la paupérisation du quartier Kénogami constituent malheureusement des faits avérés en 2011. Les indicateurs de toutes sortes confirment aisément ce constat de même que les visites de terrain. La trajectoire difficile que le quartier a empruntée n'est pas unique. Celle-ci est le reflet du développement urbain des dernières décennies: mouvements de population vers la banlieue pavillonnaire, création des centres d'achats, désindustrialisation, diminution de la taille des ménages, etc. Néanmoins, les différentes stratégies et interventions en revitalisation urbaine soulignent la volonté de divers groupes d'acteurs de travailler à l'amélioration de la situation. À cet égard, la description et l'analyse de trois types de démarches que nous avons effectuées nous renseignent sur les défis et dilemmes associés à la revitalisation urbaine dans Kénogami.

En premier lieu, la mise en valeur patrimoniale est un élément fort positif. Relier le concept de patrimoine au nom Kénogami peut être perçu comme le point de départ d'une revalorisation symbolique du milieu. Cette démarche pourrait éventuellement s'assurer d'une valorisation foncière, source d'investissements immobiliers. Cependant, le legs des premiers bâtisseurs de Kénogami est moins impressionnant que celui d'Arvida aux points de vue architectural et urbanistique.

Le concept d'aménagement y est moins spectaculaire et beaucoup plus réduit en taille. De plus, les visites guidées interpellent davantage les visiteurs que les résidants et celles-ci attirent peu de retombées économiques, du moins jusqu'à maintenant. Malgré tout, le réaménagement du Parc Price, qui s'inscrit dans ce mouvement, dote le quartier d'un espace vert majeur. Ce projet a été financé par la Ville de Saguenay et le gouvernement fédéral.
Deuxièmement, la stratégie de développement commercial de Kénogami est une démarche nécessaire. Toutefois, la qualité et la diversité des produits et services offerts sur une artère commerciale est souvent le reflet du milieu socioéconomique dans lequel elle baigne. En ce sens, les possibilités de développement commercial restent limitées, à moins d'une amélioration notable des conditions de vie des populations ou de viser une clientèle de l'extérieur. L'amélioration du cadre physique et la bonification de la gamme de commerces pourraient sans doute permettre de réduire les fuites commerciales et attirer des clients de passage. Malgré tout, la démarche traditionnelle en revitalisation a depuis longtemps démontré ses faiblesses, car l'action ne met pas l'accent sur les véritables causes du problème de dévitalisation, c'est pour cela qu'il resurgit après quelques années. De plus, cette approche conduit à un dialogue entre l'association des commerçants et la municipalité, dialogue qui tend à écarter le citoyen.

En troisième lieu, le développement communautaire ou local présente plusieurs originalités. Il vise à intégrer les citoyens dans le processus de réflexion et de décision. Il tente d'intervenir en profondeur sur le substrat socio-territorial à travers une démarche participative et la mise en valeur des ressources et potentiels locaux. Plus long et plus difficile, le développement communautaire est souvent perçu comme un pis-aller par les intervenants économiques traditionnels. Dans Kénogami, la mobilisation sociale s'est heurtée aux nombreux problèmes que vivent les populations défavorisées: manque de temps, manque de confiance en soi, sentiment d'impuissance face aux enjeux socioéconomiques, etc. Paradoxalement, les démarches de la Société d'intervention urbaine ont néanmoins relancé la concertation interne chez les commerçants.

La démarche traditionnelle en revitalisation a depuis longtemps démontrée ses faiblesses, car l'action ne met pas l'accent sur les véritables causes du problème de dévitalisation c'est pour cela qu'il resurgit après quelques années. 
En définitive, la synergie de l'ensemble des partenaires dans un milieu donné apparaît très difficile à concrétiser et l'exemple de Kénogami tend à confirmer cette réalité. L'intégration des diverses approches apparaît donc essentielle. Par ailleurs, le rôle des élus locaux est ambigu dans ce genre de processus. En effet, ceux-ci perçoivent souvent les comités de citoyens comme des lieux de contestations et de contrepouvoir qui sont en concurrence avec leur propre action. De plus, les divers gestes posés par les administrations municipales paraissent guidés principalement par le désir de visibilité des acteurs politiques, dans plusieurs cas. En cela, l'exemple de Kénogami n'est pas différent de celui des autres quartiers anciens de Saguenay ou d'ailleurs. Malgré les difficultés, la mobilisation des différents acteurs dans un projet de quartier signifiant demeure toujours un objectif important qui doit être au centre des préoccupations des acteurs socioéconomiques et politiques. Il s'agit d'un gage d'efficacité et non d'un geste vertueux!

\section{BIBLIOGRAPHIE}

${ }^{1}$ Martin Simard est géographe et urbaniste. Il est professeur titulaire à l'Université du Québec à Chicoutimi et membre du Centre de recherche sur le développement territorial.

${ }^{2}$ Suzanne Tremblay est sociologue. Elle est professeure substitut à l'Université du Québec à Chicoutimi et membre du Centre de recherche sur le développement territorial.

${ }^{3}$ Carl Brisson est géographe. Il agit comme professionnel de recherche au Laboratoire d'expertise et de recherche en géographie appliquée de l'Université du Québec à Chicoutimi.

${ }^{4}$ Divay, G. (2004). « La revitalisation urbaine intégrée. L'art de réussir un puzzle complexe », Urbanité, vol. 3, n $\mathrm{n}^{\mathrm{O}} 3,15-17$.

${ }^{5}$ Bouchard, R. (1997). Histoire de Jonquière, Cour industriel du Saguenay-Lac-Saint-Jean, Chicoutimi, p. 106.

${ }^{6}$ Côté, D. (2007). Kénogami : camériste de l'industrie, 1912-1975, Saguenay, Société historique du Saguenay, Publication $n^{\circ} 52,159$ pages.

${ }^{7}$ Leroux, G. (1972). L'expansion urbaine de Jonquière et de Kénogami en 1970-1971, Chicoutimi, Mémoire de baccalauréat en géographie, UQAC.

${ }^{8}$ Simard, M. et Goulet, A. (2005). « La question identitaire lors des débats sur les regroupements municipaux de 1975-1976 au Saguenay », Saguenayensia, vol. 47, nº 1, 143-149.
${ }^{9}$ Gaudreault, D. (1982). Plan directeur d'urbanisme, Ville de Jonquière.

${ }^{10}$ PAQUIN RECHERCHE ET ASSOCIÉS (2005). Étude de potentiel de marché et de localisation, Artères commerciales traditionnelles, Ville de Saguenay, 327 pages.

${ }^{11}$ Proulx, M.-U. et al. (2010). Le complexe Jonquière dans l'industrie de l'aluminium, Rapport final, Saguenay, Centre de recherche sur le développement territorial, UQAC, 546 pages.

${ }^{12}$ STATISTIQUE CANADA, Recensements de la population.

${ }^{13}$ Perron, M., Richard, L. et Veillette, S. (1997). «Structure sociorésidentielle et conditions de vie au Saguenay », Cahiers de géographie du Québec, vol.41, $\mathrm{n}^{\mathrm{o}} 112,31-48$.

${ }^{14}$ www.sirwilliamprice.com/historique.html.

${ }^{15}$ Tremblay, S. (2010). Les conditions d'émergence et l'ancrage territorial des entreprises d'économie sociale au Saguenay-Lac-Saint-Jean, Cahier $d u$ Réseau québécois de recherche partenariale en économie sociale, $\mathrm{N}^{\mathrm{o}}$ RQ-02-2010, Montréal, 80 pages.

${ }^{16} \mathrm{http}: / / \mathrm{www} . v i l l e . s a g u e n a y . q c . c a /$ industriecommerce xviii

${ }^{17} \mathrm{La}$ Société d'intervention urbaine ChicoutimiJonquière a fermé ses portes en 2010 à la suite du retrait du financement du gouvernement fédéral.

${ }^{18}$ Girard, P. (2005). Une levée d'idées pour un Kénogami en Chantier. Bilan général, Société d'intervention urbaine.

${ }^{19}$ Tremblay, S. (2009). " Interventions de quartier, recomposition sociale des communautés et reconversion du développement ", Économie et Solidarités, vol. $38, \mathrm{n}^{\circ} 1,182-192$. 


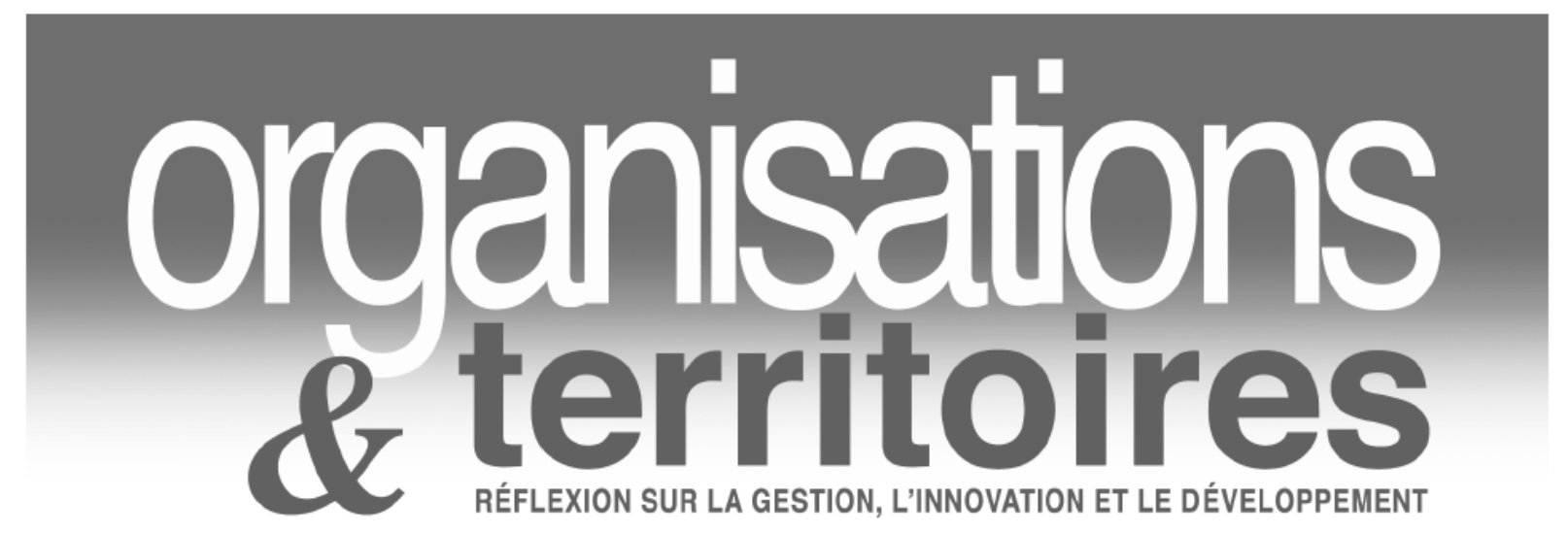

Site Web

www.ucac.ca/revueot

www.uqac.ca/revueot

www.uqac.ca/revueot

www.uqac.ca/revueot

Nous vous invitons à l'explorer et à nous faire parvenir vos commentaires et suggestions afin d'en améliorer le contenu et la présentation. 